\title{
Focal Gray Matter Changes in Schizophrenia across the Course of the Illness: A 5-Year Follow-Up Study
}

\author{
Neeltje EM van Haren",', Hilleke E Hulshoff Pol', Hugo G Schnack', Wiepke Cahn', René CW Mandl', \\ D Louis Collins', Alan C Evans ${ }^{2}$ and René S Kahn' \\ 'Department of Psychiatry, Rudolf Magnus Institute of Neuroscience, University Medical Center Utrecht, Utrecht, The Netherlands; \\ ${ }^{2}$ The Montreal Neurological Institute, McGill University, Montreal, QC, Canada
}

\begin{abstract}
Recent volumetric magnetic resonance imaging (MRI) studies have suggested brain volume changes in schizophrenia to be progressive in nature. Whether this is a global process or some brain areas are more affected than others is not known. In a 5-year longitudinal study, MRI whole brain scans were obtained from 96 patients with schizophrenia and I 3 matched healthy comparison subjects. Changes over time in focal gray and white matter were measured with voxel-based morphometry throughout the brain. Over the 5-year interval, excessive decreases in gray matter density were found in patients in the left superior frontal area (Brodmann areas 9/I0), left superior temporal gyrus (Brodmann area 42), right caudate nucleus, and right thalamus as compared to healthy individuals. Excessive gray matter density decrease in the superior frontal gray matter was related to increased number of hospitalizations, whereas a higher cumulative dose of clozapine and olanzapine during the scan interval was related to lesser decreases in this area. In conclusion, gray matter density loss occurs across the course of the illness in schizophrenia, predominantly in left frontal and temporal cortices. Moreover, the progression in left frontal density loss appears to be related to an increased number of psychotic episodes, with atypical antipsychotic medication attenuating these changes.

Neuropsychopharmacology (2007) 32, 2057-2066; doi:I 0. I038/sj.npp. I 301347; published online 28 February 2007
\end{abstract}

Keywords: schizophrenia; MRI; follow-up; voxel-based morphometry; frontal lobe; antipsychotic medication

\section{INTRODUCTION}

Schizophrenia is a severe and disabling psychiatric illness, affecting approximately $1 \%$ of the population. It is characterized by a heterogeneous course, often with clinical deterioration and poor outcome. So far, the etiology of the illness remains largely unknown, although genetic and environmental factors appear to be causally involved (Mueser and McGurk, 2004). Brain abnormalities have been hypothesized to be present in schizophrenia from the first delineation of the illness by Kraepelin, who postulated frontal- and temporal-lobe abnormalities to be central to the pathology of the disease (Kraepelin, 1919). Indeed, recent post-mortem (Selemon et al, 2003) as well as crosssectional region of interest (Hulshoff Pol et al, 2002) and statistical parametric mapping studies (Hulshoff Pol et al, 2001; Kubicki et al, 2002; Wright et al, 1999) have provided a considerable amount of evidence for brain volume

\footnotetext{
*Correspondence: Dr NEM van Haren, Department of Psychiatry, HP. A.01.126, Rudolf Magnus Institute of Neuroscience, University Medical Center Utrecht, Heidelberglaan 100, 3584 CX Utrecht, The Netherlands, Tel: + 3130250 7I30, Fax: + 31302505443 ,

E-mail: N.E.M.vanHaren@umcutrecht.nl

Received 9 June 2006; revised 2 January 2007; accepted 3 January 2007
}

abnormalities in frontal and temporal regions of the brain in schizophrenia.

Schizophrenia is often characterized as a progressive illness based on the deterioration in functioning in these patients (Mueser and McGurk, 2004). Progressive decreases in global gray matter volume have been found in adolescents with childhood-onset schizophrenia (Sporn et al, 2003) and in schizophrenia patients in the early (Cahn et al, 2002; Wood et al, 2001) and chronic (Mathalon et al, 2001) stages of the disease. However, it is unclear whether the progressive loss of brain tissue is a global phenomenon or whether the tissue loss over time is indeed more pronounced in focal brain areas. Several longitudinal studies examined specific brain areas, such as frontal lobe (Ho et al, 2003), superior temporal gyrus (Kasai et al, 2003a), and Heschl's gyrus or planum temporale (Kasai et al, 2003b) and found these volumes to decrease progressively over time in schizophrenia patients. However, it is unknown whether the local changes are limited to these frontal and temporal areas or are more widespread across the brain. Statistical parametric mapping approaches, such as voxel-based morphometry (VBM) have several advantages over the standard volume measurements: it is not biased to one particular structure, includes structures that are difficult to quantify by the standard volume measurements, and provides a comprehensive assessment of 
anatomical differences throughout the brain (Ashburner and Friston, 2000). The current study sets out to investigate focal brain changes in patients with schizophrenia relative to healthy subjects across the adult age range, examining the whole brain in a 5-year longitudinal study using VBM.

\section{PATIENTS AND METHODS}

\section{Subjects}

A 5-year follow-up magnetic resonance imaging (MRI) study was carried out, including patients with schizophrenia and healthy comparison subjects. At baseline (T0), 159 patients and 158 healthy individuals were scanned (Hulshoff Pol et al, 2001). A total of 96 patients (70 male/26 female) and 113 comparison subjects ( 76 male/37 female) completed the longitudinal study and were rescanned after an interval of 5 years (T5). The study was approved by the Humans Ethics Committee of the University Medical Center Utrecht. Written informed consent was obtained for all subjects.

At follow-up, clinical assessment was similar to what had been carried out at baseline and has been described in detail by Hulshoff Pol et al (2001). Therefore, the clinical assessment is described only briefly. Subjects with a major medical or neurological illness or an IQ below 80 were excluded. Both at baseline and follow-up, the presence or absence of psychopathology was established by using the Comprehensive Assessment of Symptoms and History (CASH; Andreasen et al, 1992) and was assessed by two independent raters. Patients gave permission to contact their treating physician or nurse for further information and medical records were used when necessary. In case the information provided by the patient, medical records, treating physician, or nurse was not reliable, the patient was excluded from the analysis. Diagnostic consensus was achieved in the presence of a psychiatrist. All patients met DSM-IV criteria for schizophrenia or schizophreniform disorder at time of first measurement; those with schizophreniform disorder were reassessed and met the criteria for a diagnosis of schizophrenia after 1 year of illness. At follow-up, all patients met criteria for schizophrenia except four patients who received a diagnosis of schizoaffective disorder. Three patients received the additional DSM-IV diagnosis of drug abuse or dependence at follow-up. Severity of illness was measured with the Positive and Negative Syndrome Scale (PANSS; Kay et al, 1987). All healthy comparison subjects met Research Diagnostic Criteria (Pfohl et al, 1995) for 'never mentally ill' and had no first-degree family members with a psychotic illness.

The comparison subjects were matched for age, sex, handedness, height, socioeconomic status of their parents (expressed as the highest level of education completed by one of the parents), and follow-up duration. For demographic information see Table 1. Reasons subjects (63 patients/45 controls) did not participate in the follow-up study are summarized in Table 2.

A proportion of the subjects who participated in the cross-sectional study could not be included at follow-up. At baseline, the patients and comparison subjects included in the follow-up were younger than the patients $(32.22 \mathrm{vs} 39.95$ years; $p<0.001)$ and comparison subjects (35.28 vs 43.41 years; $p=0.001$ ) who failed to complete follow-up. The patients included at follow-up had a shorter 'duration of illness' (10.95 vs 19.85 years; $p<0.001)$ and fewer negative symptoms (mean total rating on PANSS negative symptom scale of 17.79 vs $20.05 ; p=0.032$ ) than the patients who did not complete the follow-up.

Scan interval was not significantly different between the groups (patients: 4.83 (0.55) years; controls: $4.94(0.32)$ years). The duration of the scan interval did not correlate significantly with age in either of the groups.

To calculate the cumulative dosage of typical antipsychotics during the scan interval, a table from the Dutch National Health Service was used to derive the haloperidol equivalents (Commissie Farmaceutische Hulp van het College voor Zorgverzekeringen, 2002). For atypical antipsychotics, the respective pharmaceutical companies suggested conversion rates into haloperidol equivalents (clozapine, 40:1; olanzapine, 2.5:1; risperidone, 1:1; sulpiride, $170: 1$; quetiapine, $50: 1$; and sertindole, $2: 1$ ). No reliable information on medication intake during the scan interval was available for six patients. Ten patients had been taking typical antipsychotic medication exclusively and 27 patients atypical antipsychotic medication exclusively over the entire 5-year period. Forty-three patients switched between typical and atypical medication during the scan interval. Thirty out of these 43 patients switched from typical to atypical antipsychotic medication, one patient from atypical to typical antipsychotic medication and 12 patients changed several times between the two types of drug during the 5-year interval. Clozapine and olanzapine were the types of atypical antipsychotics most often prescribed.

'Age at onset of illness' was defined as the first time the patients experienced psychotic symptoms, as obtained from the CASH interview (Andreasen et al, 1992). Duration of illness was defined as time between age at onset of illness and age at first MRI scan. Information on number of hospitalizations and total duration of hospitalization during the scan interval was obtained from the CASH interview. It is estimated that $90 \%$ of the hospitalizations of schizophrenia patients in The Netherlands (and also in this cohort) is a consequence of psychotic relapses.

\section{Brain Imaging}

Magnetic resonance images were acquired on a Philips NT scanner operating at $1.5 \mathrm{~T}$ in all subjects. The acquisition protocol for the T1- and T2-weighted images and the preprocessing of the scans has been described in detail by Hulshoff Pol et al (2001).

Intensity histogram analysis yielded thresholds for separating brain tissue from cerebrospinal fluid and, within the brain, gray matter from white matter. Gray and white matter segments were created by applying these thresholds to the images (Schnack et al, 2001). The segments were blurred by a 3D Gaussian kernel (full-width at halfmaximum $(\mathrm{FWHM})=8 \mathrm{~mm}$ ) to conform the data to the Gaussian field model underlying the statistical procedures used for making inferences about significance. The voxel values of these blurred gray and white matter segments reflect the local presence, or concentration, of gray or white matter, respectively, and these images are referred to as 'density maps'. 
Table I Demographic Information (N or Mean (SD)) at Inclusion (T0) and Follow-Up (T5) of Patients with Schizophrenia $(n=96)$ and Comparison Subjects $(n=113)$

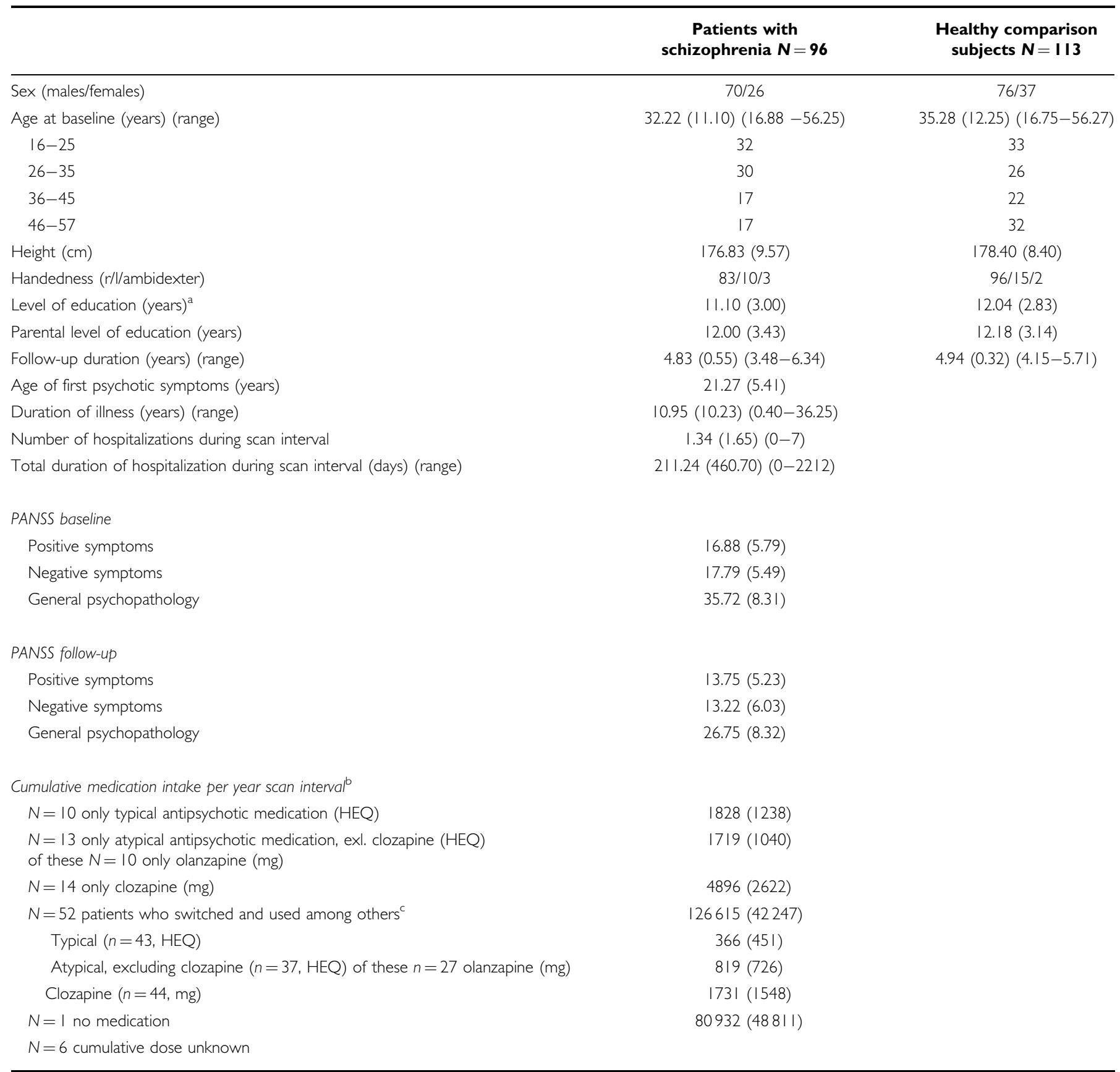

aLevel of education was significantly lower in patients than in comparison subjects $(p<0.05)$.

${ }^{b}$ Cumulative typical and atypical antipsychotic medication intakes are in haloperidol equivalents (HEQ) per year scan interval. Cumulative clozapine and olanzapine intake are in milligram (mg) per year scan interval.

'During the scan interval, 52 patients switched between at least two of typical antipsychotics, atypical antipsychotics, and clozapine. For example, 43 of these patients used typical antipsychotic medication at some point during the scan interval, but have also been taken atypical antipsychotics and/or clozapine.

To compare T0-T5 differences in brain tissue at the same anatomical locations in all subjects, we have to transform maps of the within-subject differences in density into a standard coordinate system. This was done in the following way. First, the T0 images were linearly transformed to the model brain, the previously determined 'most average' brain (Hulshoff Pol et al, 2001). In this linear step, a joint entropy mutual information metric was optimized. In the second step, nonlinear (elastic) transformations were calculated to warp the linearly transformed T0 images to the model brain using the ANIMAL (Collins et al, 1995) program. The nonlinear registration sampling and stiffness parameters were chosen to minimize global shape differences between brains, although retaining most of local 
Table 2 Information on Why Subjects did not Participate at Follow-up

\begin{tabular}{|c|c|c|}
\hline Reasons not to participate & $\begin{array}{l}\text { Patients with } \\
\text { schizophrenia }\end{array}$ & $\begin{array}{l}\text { Healthy comparison } \\
\text { subjects }\end{array}$ \\
\hline Refusal to take part & 26 & 18 \\
\hline $\begin{array}{l}\text { Recently developed medical condition (ie installation of pacemaker, } \\
\text { major bypass surgery, cancer treatment, meningitis) }\end{array}$ & 2 & 5 \\
\hline Too ill to participate & 16 & \\
\hline Died & 2 & \\
\hline Poor scan quality (no separation of gray and white matter possible) & 14 & 4 \\
\hline Older than 58 years of age (no patients in this age range) & - & 8 \\
\hline
\end{tabular}

differences. Sampling was limited to a scale of $4 \mathrm{~mm}$ (FWHM), with deformation vectors estimated every $2 \mathrm{~mm}$ throughout the volume. Stiffness was kept high to have a smooth transformation, thus minimizing changes to local volume. Registration with these parameters corrected for overall brain shape without significantly modifying local dimensions, such as cortical thickness. To allow for a maximal comparability between the T0 image and T5 image within subjects, a rigid linear transformation of T5-T0 was computed for each subject. The linear transformation from a subject's T5-T0 image and the linear and nonlinear transformations from the T0 image to the standardized brain were concatenated. The gray and white matter density maps of the T0 and T5 images were transformed to the standardized coordinate system by applying the total transformation. Gray and white matter density difference maps were calculated by subtracting each subject's density map at T0 from the density map at T5. Finally, the density difference maps were resampled to voxels of size $2 \times 2 \times 2.4 \mathrm{~mm}^{3}$.

\section{Statistical Analysis}

To investigate differences in change in focal density between groups during the scan-interval linear regression, analysis was performed through all brains for each voxel separately in the gray and white matter density difference maps. Group (patients, comparisons), sex (male, female), and handedness (right, left/ambidexter) entered the analysis as predictor variables; age at baseline served as covariate. To evaluate interaction with age, a similar linear regression analysis was performed, adding the interaction between age and group as a predictor variable.

Given the number of subjects $(n=209)$, number of scans per subject $(n=2)$, FWHM of the blur kernel $(8 \mathrm{~mm})$, voxel size $\left(2 \times 2 \times 2.4 \mathrm{~mm}^{3}\right)$, and volume of the search region $\left(1350 \mathrm{~cm}^{3}\right)$, the critical threshold $t$-value for a two-tailed $\alpha$ significance level of 0.05 after correcting for multiple comparisons is $|t|>5.05(\mathrm{df}=205)$, according to random field theory (Worsley et al, 1996).

In case of significant main effects in density changes during the scan interval in schizophrenia, several post hoc analyses were done. The values of the voxels showing excessive change in patients were extracted and multiple linear regression analyses were performed on each voxel using SPSS. Sex (male, female) and handedness (right, left/ ambidexter) entered the analyses as predictor variables; age served as covariate. The influence of the following variables on this excessive change in density in patients was examined by adding them as predictor variables into the regression analysis: (1) the interaction between group and sex, (2) number of hospitalizations during the scan interval or total duration of hospitalization during the scan interval, (3) total typical or atypical antipsychotic medication intake in haloperidol equivalents per year scan interval, (4) total clozapine or olanzapine intake in milligram per year scan interval, and (5) lateral and third ventricular volumes.

Number and duration of hospitalizations and most cumulative medication intake variables were not normally distributed. This is not problematic in regression analysis as long as the residuals are normally distributed. Therefore, unstandardized residuals were saved and examined for normality of the distribution. However, for total amount of typical medication, one patient was considered an outlier, because of the high dose compared to other patients. Therefore, in the case of significant findings on cumulative typical medication intake, the analysis was repeated without this patient.

To investigate, the influence of duration of illness is complicated for two reasons. A group comparison is not possible and secondly, duration of illness is highly correlated with age $(r=0.88 ; p<0.001)$ leading to the problem of multicolinearity.

\section{RESULTS}

For illustrations purposes only, Figure 1 shows the group effect of decreased gray matter density in patients with schizophrenia as compared to healthy individuals at baseline and at follow-up.

\section{Longitudinal Data}

During the 5-year interval, excessive decreases in gray matter density were found in patients with schizophrenia in the left superior frontal gyrus (medial aspect: Brodmann areas 9/10), left superior temporal gyrus (Brodmann area 
a

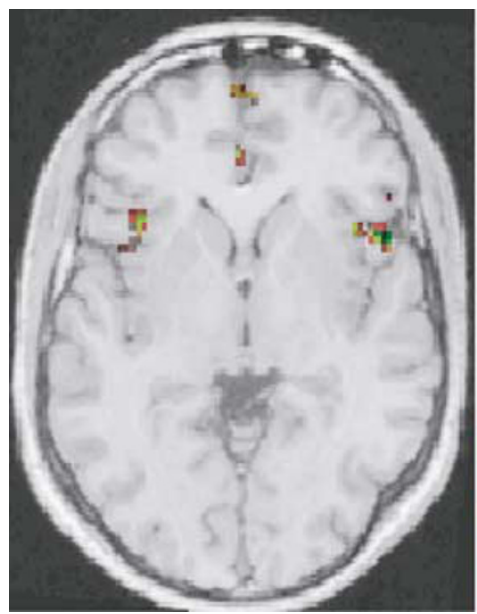

at TO: Talairach coordinate (axial): $z=3$ b

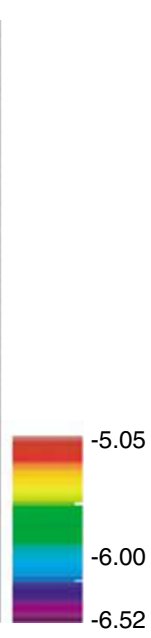

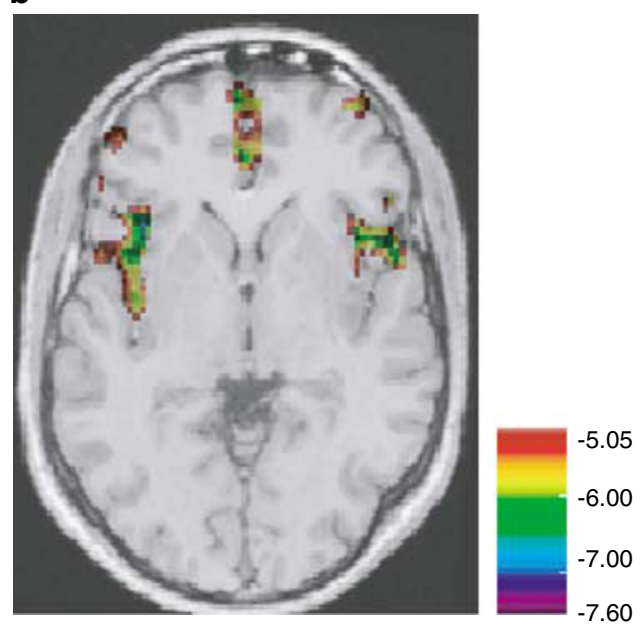

at T5: Talairach coordinate (axial): $z=3$

Figure I Cross-sectional analysis: Focal decreases in gray matter density in patients with schizophrenia as compared to healthy individuals at baseline (a) and follow-up (b). The thresholded map of $t$-statistic values $(t<-5.05)$ is superimposed on an axial $(z=3)$ section through the magnetic resonance image of the model brain. The $z$ value indicates the distance (in millimeter) of a given section from axial plane passing through the anterior commissure.

Table 3 Excessive Gray Matter Density Change in Patients with Schizophrenia Relative to Comparison Subjects during the Scan Interval

Excessive density change in

Talairach coordinates $(\mathrm{mm})$ schizophrenia during scan interval at $|t|>5.05$

\begin{tabular}{|c|c|c|c|c|c|}
\hline \multirow[b]{2}{*}{ Brain area } & & \\
\hline & $x$ & y & $\mathbf{z}$ & b & $t$ \\
\hline \multicolumn{6}{|l|}{ Left hemisphere } \\
\hline \multirow[t]{2}{*}{ Superior frontal gyrus (Brodmann area $9 / 10$ ) } & -7 & 58 & 25 & -0.029 & -5.54 \\
\hline & -21 & 51 & 31 & -0.031 & -5.07 \\
\hline Superior temporal gyrus (Brodmann area 42) & -67 & -24 & 17 & -0.024 & -5.12 \\
\hline \multirow[t]{2}{*}{ Caudate nucleus } & 13 & -2 & 27 & -0.029 & $-5.7 \mid$ \\
\hline & 11 & 10 & 17 & -0.036 & -5.06 \\
\hline Thalamus & 7 & -31 & 13 & -0.025 & -5.12 \\
\hline
\end{tabular}

The Talairach coordinates $x, y$, and $z$ indicate the distance (in millimeters) of a given section from the sagittal, coronal, and axial plane, respectively, passing through the anterior commissure. Regression coefficient $(b)$ represents the excessive density change at Talairach coordinates $(x, y, z)$ in patients with schizophrenia relative to comparison subjects during the scan interval. $t$ represents the $t$-statistic values at the Talairach coordinates.

42), right caudate nucleus, and right thalamus as compared to healthy individuals (Table 3, Figure 2).

The coordinates presented in the table are the peak values in the clusters of voxels with a threshold $|t|>5.05$. The caudate nucleus consists of 23 voxels, whereas the thalamus consists of only 3 voxels. The superior frontal gyrus has 33 voxels above the threshold and the superior temporal gyrus 4 voxels. The figures are presented at the significance threshold $(t=5.05)$.

No significant differences in change in white matter density during the scan interval between patients and comparison subjects were found. No significant ageby-group and sex-by-group interactions were found for gray and white matter density changes during the scan interval.
Excluding the three patients that were abusing or dependent on drugs during the scan interval did not change the findings.

\section{Outcome, Medication}

Post hoc analyses on the significant voxels revealed that number of hospitalizations during the scan interval was significantly associated with a larger decrease in gray matter density in the left superior frontal gyrus (Table 4). Total duration of hospitalization during the scan interval could not explain the excessive changes in gray matter density in patients.

Cumulative clozapine and olanzapine intake per year scan interval was significantly related to less decrease in density 
a

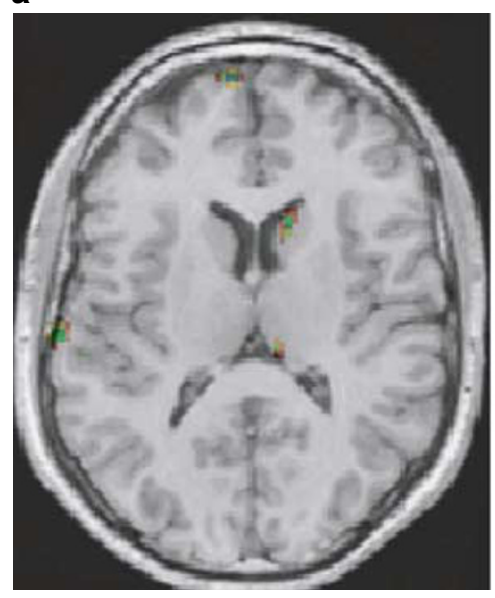

Talairach coordinate (axial): $z=15$ b

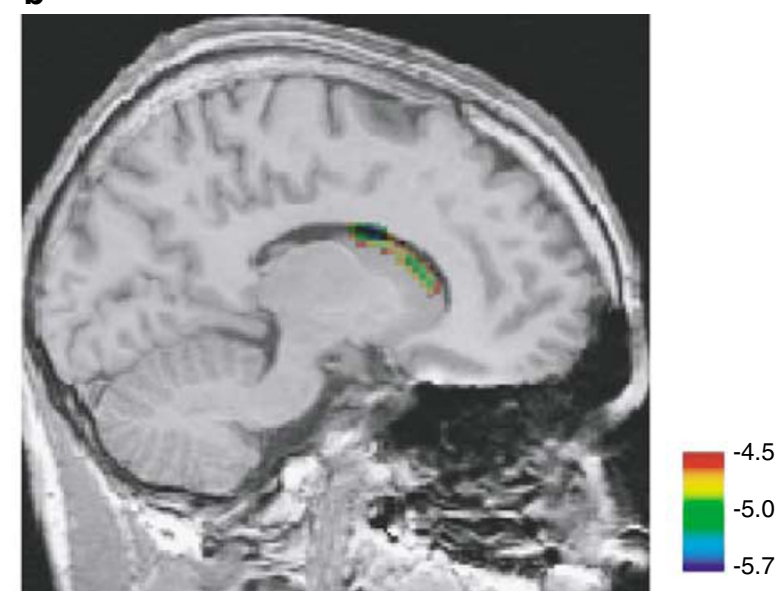

Talairach coordinate (sagittal): $x=11$

Figure 2 Longitudinal analysis: Excessive gray matter density decrease during the scan interval in patients with schizophrenia as compared to healthy individuals. The thresholded map of $t$-statistic values $(t<-4.5)$ is superimposed on an axial $(a, z=15)$ and sagittal $(b, x=1 \mathrm{I})$ section through the magnetic resonance image of the model brain. $z$ and $x$ values indicate the distance (in millimeter) of a given section from the sagittal and axial plane, respectively, passing through the anterior commissure.

Table 4 Association between Excessive Decreases in Gray Matter Density Relative to Healthy Individuals and Cumulative Medication Intake per Year Scan Interval and Number of Hospitalizations during the Scan Interval in Patients with Schizophrenia

Talairach coordinates $(\mathrm{mm})$

\begin{tabular}{|c|c|c|c|c|c|c|c|}
\hline Brain area & $x$ & $y$ & $\mathbf{z}$ & Clinical measure (calculated over scan interval) & $\boldsymbol{b}^{\mathrm{a}}$ & $t$ & $p$ \\
\hline \multicolumn{8}{|l|}{ Left hemisphere } \\
\hline \multirow[t]{3}{*}{ Superior frontal gyrus } & -7 & 58 & 25 & Cumulative olanzapine dose/year ${ }^{\text {b }}$ & $5.53 E-06$ & 2.20 & 0.05 \\
\hline & & & & Cumulative olanzapine dose/year ${ }^{b}$ & $7.59 E-06$ & 2.63 & 0.0 \\
\hline & & & & Number of hospitalizations & $-6.01 \mathrm{E}-03$ & -2.21 & 0.0 \\
\hline
\end{tabular}

Right hemisphere

Caudate nucleus

$13-2$

-2
10

27

Cumulative typical antipsychotic dose/year ${ }^{c}$

$2.22 \mathrm{E}-05$

2.29

0.03

II

17 Cumulative typical antipsychotic dose/year ${ }^{c}$

$3.42 \mathrm{E}-05$

2.19

0.03

${ }^{a}$ Regression coefficient (b) represents the change in gray matter density in case of an increase with I unit in the clinical measure. For example, an increase of 1000 mg of clozapine per year scan interval is related to an increase in gray matter density in the superior frontal gyrus $(x=-7, y=58, z=25)$ with $1000 \times 5.53 \mathrm{E}-06=0.0053$. A positive $b$ value for the superior frontal gyrus here reflects a less excessive density decrease in patients relative to comparison subjects.

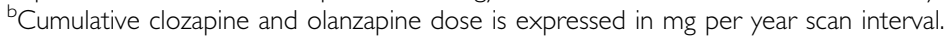

${ }^{c}$ Cumulative typical antipsychotic dose is expressed in haloperidol equivalents per year scan interval. One patient who was using a high dose of typical antipsychotic medication was excluded from the analysis.

in the left superior frontal gyrus (Figure 3). Because this same brain area was also related to total number of hospitalizations, we adjusted the analysis for this factor. The findings did not change after correction for number of hospitalizations.

Cumulative typical antipsychotic medication per year scan interval was significantly associated with increased density in the right caudate nucleus and thalamus (at trend level) and left superior frontal gyrus. However, exclusion of one patient who was prescribed a large dose compared to all other patients revealed that the association with the thalamus and superior frontal gyrus no longer reached significance (Table 4).

The saved unstandardized residuals from the post hoc analyses that reached significance revealed normal distributions of the data (Kolmogorov-Smirnov statistic $=$ not significant).

Lateral and third ventricular volumes did not explain the differences between groups in any of the significant gray matter density changes over time despite significant correlations with the significant gray matter density changes (explaining approximately $25 \%$ of the variance in both patients and controls). 

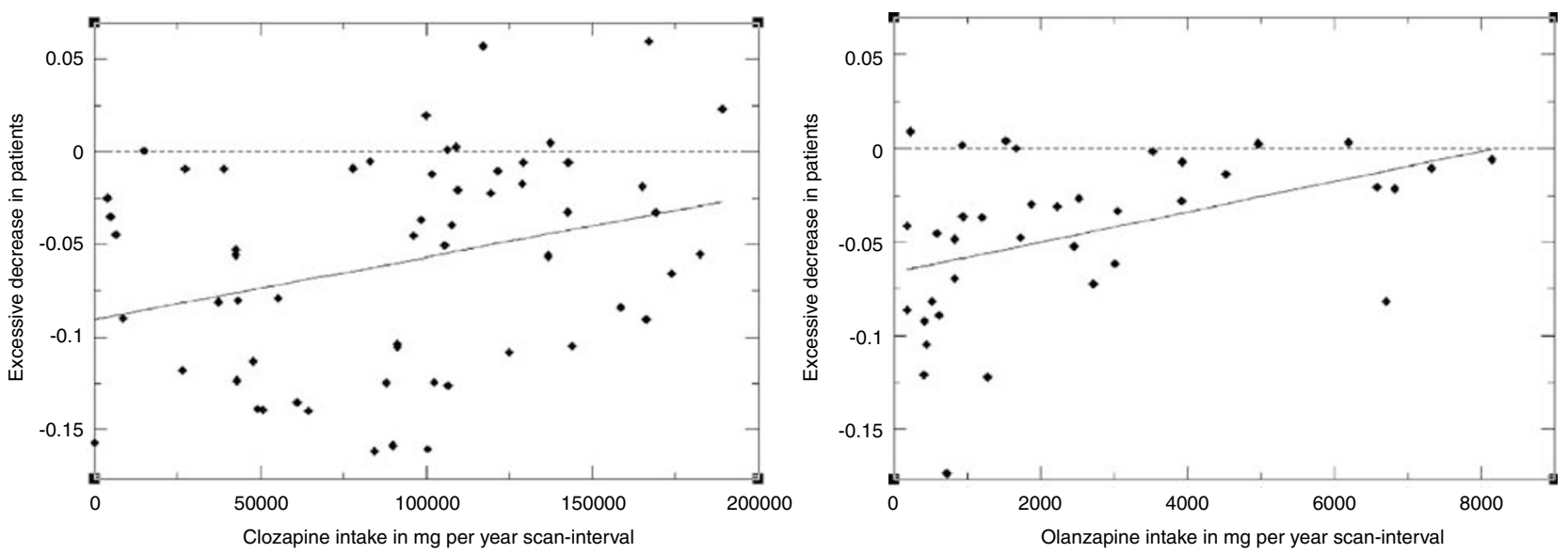

Figure 3 Relationship between cumulative intake of clozapine and olanzapine in mg per year scan interval and excessive decrease in density in the superior frontal gyrus in patients with schizophrenia relative to healthy individuals. The $x$-axis represents the intake of clozapine or olanzapine (mg) per year scan interval. The $y$-axis represents the unstandardized residuals of the densities in the superior frontal lobe after regressing out the factors common in patients and controls (age, gender, and handedness), that is, leaving only the effect of group. The regression line indicates that with increasing medication intake, the patient's superior frontal gray matter density change is more similar to that of the comparison subjects $(y=0$, dashed line).

\section{DISCUSSION}

In this longitudinal study, focal brain changes were examined across the course of the illness in patients with schizophrenia. Gray and white matter density changes were compared between 96 patients with schizophrenia and 113 healthy individuals over a 5-year interval using VBM. The main findings of this study are two-fold. First, excessive decreases in gray matter density were found in specific areas of the brain, that is, left superior frontal gyrus (Brodmann area 9/10), left superior temporal gyrus (Brodmann area 42), right caudate nucleus, and right thalamus in patients with schizophrenia as compared to healthy subjects. Second, the density changes in the frontal lobe were most pronounced in patients with the poorest course of the illness (expressed as number of hospitalizations during the scan interval). Conversely, treatment with clozapine and olanzapine (expressed as cumulative dose per year scan interval), but not treatment with typical antipsychotics, attenuated the loss of gray matter density in the same frontal area. This effect remained significant after adjustment for the number of hospitalizations during the interval.

The loss of brain tissue over time was localized in those brain areas that have been found to be affected in schizophrenia in cross-sectional neuroimaging (Hulshoff Pol et al, 2001, 2002; Kubicki et al, 2002; Wright et al, 1999) as well as in post-mortem studies (Harrison, 1999). Indeed, the area where loss of gray matter density was most pronounced in our study (Brodmann area 9) is the same as was reported to be affected in earlier post-mortem studies (Selemon et al, 1995, 2003). Interestingly, in post-mortem studies it was suggested that there was tissue loss in Brodmann area 9 secondary to loss of neuropil, not to a diminished number of neuronal cells (Selemon et al, 1995, 2003). Possibly, this gray matter tissue loss in the frontal cortex is of a progressive, not a static nature. Furthermore, the finding that the excessive decrease in gray matter density in this brain area was related to a higher number of hospitalizations during the scan interval suggests these changes to be related to the pathogenesis of the disease. Because hospitalizations are most often (though by no means exclusively) related to psychotic relapses, the loss of gray matter density may have resulted from the hypothesized toxic effect of the psychotic state (Arango et al, 2001) or these patients showed more recurrent episodes of poor self care. In addition, independently of number of hospitalizations, cumulative antipsychotic treatment attenuated the decrease in gray matter density in the same frontal area. Interestingly, this effect was attributable to the atypical antipsychotics (clozapine and olanzapine) and was not seen with the use of typical antipsychotics, such as haloperidol and pimozide. This finding is consistent with that of a study comparing the effects of olanzapine and haloperidol on gray and white matter volumes in firstepisode schizophrenia over a 1-year period, reporting progressive (global) gray matter loss in the patients on haloperidol, but not in those treated with olanzapine (Lieberman et al, 2005). It is of note that this effect was observed both in first-episode patients (Lieberman et al, 2005) as well as in the more chronically ill cohort that we studied, suggesting that atypical antipsychotics may have beneficial effects on the brain even in the more chronic stages of the illness. However, it cannot be excluded that the association described here may actually concern the release from exposure to typical antipsychotics, because a proportion of the patients changed from typical to olanzapine or clozapine during the scan interval.

At first glance, the excessive decrease in gray matter density in the caudate nucleus over time may seem at odds with earlier cross-sectional reports indicating increased caudate volume in schizophrenia (Chakos et al, 1994). However, an increase in caudate nucleus density was indeed present in patients with schizophrenia at baseline, but not at follow-up. It was shown that the excessive decrease in caudate nucleus density was smaller when patients took 
more typical antipsychotic medication per year scan interval. In our study, almost half of the patients used both typical and atypical antipsychotics during the scan interval, with the majority of these patients switching from a typical to an atypical drug. This suggests, consistent with earlier studies (Chakos et al, 1995), that caudate nucleus density decreased (or normalized) as a result of the inclusion of more patients on atypical antipsychotic medication at follow-up than at baseline.

The relationship between excessive density change in patients and the type and dose of antipsychotic exposure as examined in this study is subject to several caveats. It is not possible to rule out a selection bias (there was some evidence for a selection bias toward the inclusion of good outcome patients) or reverse causality. In addition, it should be noted that patients differed in the amount of medication that they had used before inclusion in the study, whereas reliable information was unavailable on their lifetime cumulative medication use. Furthermore, most patients changed medication during the scan interval. Also, no information was available about the compliance to the prescribed medication. The conversion rates used to convert atypical antipsychotic dose to haloperidol equivalents are by no means generally accepted. To overcome this problem, cumulative olanzapine and clozapine intake in mg per year during the scan interval was looked at separately in the primary analysis without converting them in to haloperidol equivalents. In addition, the $p$-values of these subgroup analyses only just reached significance at a 0.05 level, that is, the presence of type- 1 errors cannot be excluded.

A progressive decrease in density was found in the right posterior thalamic region in patients with schizophrenia. The thalamus has been reported to be decreased in crosssectional studies in schizophrenia (Andreasen et al, 1994), particularly in the pulvinar nuclei (Kemether et al, 2003). Post-mortem studies also found evidence for reduced volume of the pulvinar in patients with schizophrenia (Danos et al, 2003). Consistent with our findings, longitudinal studies of midsagittal thalamic areas in childhoodonset schizophrenia also show a progressive reduction over time (Kumra et al, 2000).

The fact that the excessive gray matter density loss in the superior fronto-temporal cortex was primarily left-sided is consistent with the reported left-sided progressive volume reduction in gray matter of the superior temporal gyrus, Heschl's gyrus, and planum temporale in region-of-interest studies (Kasai et al, 2003a,b) and with the postulated predominance of left-sided brain involvement in schizophrenia (Crow, 1997).

The interaction between group and age was added into the analysis to investigate whether the groups differed in age dependency of the gray matter density changes. No significant interactions were found. However, it could be argued that the density decreases are possibly larger in patients who are early in the course of the illness, that is, younger of age. It is these patients that have a higher number of hospitalizations (illness duration and number of hospitalizations: Spearman's $\rho=-0.37 p<0.001$; age and number of hospitalizations: Spearman's $\rho=-0.50$ $p<0.001)$ and number of hospitalizations during the scan interval was significantly correlated with excessive GM density decrease.
Our findings are in agreement with a longitudinal cortical mapping study in childhood-onset schizophrenia in which a dynamic pattern of accelerated cortical gray matter loss was found (Thompson et al, 2001): at disease onset parietal areas appeared to be involved, whereas temporal and frontal areas were affected later in the disease. In addition, the transition from prodromal phase into frank psychosis has been associated with (inferior) frontal and temporal decreases in gray matter density (Job et al, 2005; Pantelis et al, 2003), whereas first-episode patients showed frontotemporal gray matter density decreases during the first 2 years of illness (Farrow et al, 2005).

The results should be considered in view of the limitations of this study. First, VBM was used to assess differences in gray and white matter density between patients and controls. Methodological issues regarding this method have been discussed in the literature, including spatial normalization and statistical significance (Ashburner and Friston, 2000, 2001; Bookstein, 2001). Indeed, considering the stringent statistical corrections that are needed for multiple comparisons according to random field theory (Worsley et al, 1996) and the necessary blurring of the gray and white matter maps, some existing differences in density change between patients with schizophrenia compared to controls (and interaction effects with age) may not have been revealed. These statistical corrections are designed to minimize false positive findings so that we can be certain (as regard to the level of significance) of the regions identified; however, we cannot speak to the lack of finding in some regions.

Furthermore, the analyses were based on the assumption that brain changes are linear over time, both in patients and comparison subjects and across the age range. It could be that focal changes in gray and white matter density in the brain may develop unequally over time, that is, occur sequentially, with some areas being affected earlier than others, or that changes in certain areas occur at a particular age (Giedd et al, 1999). The reliability and validity of the classification into gray and white matter and the transformation of the individual brains to a standardized brain might be even more relevant in a longitudinal design in which follow-up and baseline scan of an individual are subtracted. Minimal differences between the two scans in separating gray and white matter or small errors in the linear transformation from the follow-up to the baseline scan introduce noise into the final statistical analyses. Consequently, detecting subtle differences between the groups may become difficult.

We chose to analyze plain differences in gray and white density. The use of corrected differences, either by dividing by the baseline (T0) value or by covarying for the T0 value, seems less appropriate to answer the question whether at a certain voxel gray or white matter presence decreases more in patients than in controls (see (Wainer, 1991) for a thorough analysis of this topic). Effects from cohort or time of measurement cannot be excluded completely. Yet it seems unlikely that they explained the findings as possible confounding effects of age were controlled for in the analysis and patients and comparison subjects were scanned intermixed over time.

In conclusion, in this study, changes in gray and white matter densities were compared between patients with 
schizophrenia and healthy comparison subjects over a 5year interval. It was found that gray matter density loss occurs across the course of the illness in schizophrenia, predominantly in left-sided frontal and temporal cortices and in the right thalamus. Moreover, the progression in left frontal density loss appeared related to an increased number of psychotic episodes, with clozapine and olanzapine possibly attenuating these changes.

\section{REFERENCES}

Andreasen NC, Flashman L, Flaum M, Arndt S, Swayze V, O’Leary DS et al (1994). Regional brain abnormalities in schizophrenia measured with magnetic resonance imaging. JAMA 272: 17631769.

Andreasen NC, Flaum M, Arndt S (1992). The Comprehensive Assessment of Symptoms and History (CASH). An instrument for assessing diagnosis and psychopathology. Arch Gen Psychiatry 49: 615-623.

Arango C, Kirkpatrick B, Koenig J (2001). At issue: stress, hippocampal neuronal turnover, and neuropsychiatric disorders. Schizophr Bull 27: 477-480.

Ashburner J, Friston KJ (2000). Voxel-based morphometry-the methods. Neuroimage 11: 805-821.

Ashburner J, Friston KJ (2001). Why voxel-based morphometry should be used. Neuroimage 14: 1238-1243.

Bookstein FL (2001). 'Voxel-based morphometry' should not be used with imperfectly registered images. Neuroimage 14: 1454-1462.

Cahn W, Hulshoff Pol HE, Lems EB, van Haren NE, Schnack HG, Van Der Linden JA et al (2002). Brain volume changes in firstepisode schizophrenia: a 1-year follow-up study. Arch Gen Psychiatry 59: 1002-1010.

Chakos MH, Lieberman JA, Alvir J, Bilder R, Ashtari M (1995). Caudate nuclei volumes in schizophrenic patients treated with typical antipsychotics or clozapine. Lancet 345: 456-457.

Chakos MH, Lieberman JA, Bilder RM, Borenstein M, Lerner G, Bogerts B et al (1994). Increase in caudate nuclei volumes of first-episode schizophrenic patients taking antipsychotic drugs. Am J Psychiatry 151: 1430-1436.

Collins DL, Holmes CJ, Peters TM, Evans AC (1995). Automatic 3D model-based neuroanatomical segmentation. Hum Brain Mapp 3: 190-208.

Commissie Farmaceutische Hulp van het College voor Zorgverzekeringen (2002). Farmacotherapeutisch Kompas (in Dutch). Amstelveen: The Netherlands.

Crow TJ (1997). Is schizophrenia the price that Homo sapiens pays for language? Schizophr Res 28: 127-141.

Danos P, Baumann B, Kramer A, Bernstein HG, Stauch R, Krell D, et al (2003). Volumes of association thalamic nuclei in schizophrenia: a postmortem study. Schizophr Res 60: 141-155.

Farrow TF, Whitford TJ, Williams LM, Gomes L, Harris AW (2005). Diagnosis-related regional gray matter loss over two years in first episode schizophrenia and bipolar disorder. Biol Psychiatry 58: 713-723.

Giedd JN, Jeffries NO, Blumenthal J, Castellanos FX, Vaituzis AC, Fernandez $\mathrm{T}$ et al (1999). Childhood-onset schizophrenia: progressive brain changes during adolescence. Biol Psychiatry 46: 892-898.

Harrison PJ (1999). The neuropathology of schizophrenia. A critical review of the data and their interpretation. Brain 122(Part 4): 593-624.

Ho BC, Andreasen NC, Nopoulos P, Arndt S, Magnotta V, Flaum M (2003). Progressive structural brain abnormalities and their relationship to clinical outcome: a longitudinal magnetic resonance imaging study early in schizophrenia. Arch Gen Psychiatry 60: 585-594.
Hulshoff Pol HE, Schnack HG, Bertens MG, van Haren NE, van der Tweel I, Staal WG et al (2002). Volume changes in gray matter in patients with schizophrenia. Am J Psychiatry 159: 244-250.

Hulshoff Pol HE, Schnack HG, Mandl RC, van Haren NE, Koning $\mathrm{H}$, Collins DL et al (2001). Focal gray matter density changes in schizophrenia. Arch Gen Psychiatry 58: 1118-1125.

Job DE, Whalley HC, Johnstone EC, Lawrie SM (2005). Grey matter changes over time in high risk subjects developing schizophrenia. Neuroimage 25: 1023-1030.

Kasai K, Shenton ME, Salisbury DF, Hirayasu Y, Lee CU, Ciszewski AA et al (2003a). Progressive decrease of left superior temporal gyrus gray matter volume in patients with first-episode schizophrenia. Am J Psychiatry 160: 156-164.

Kasai K, Shenton ME, Salisbury DF, Hirayasu Y, Onitsuka T, Spencer MH et al (2003b). Progressive decrease of left Heschl gyrus and planum temporale gray matter volume in first-episode schizophrenia: a longitudinal magnetic resonance imaging study. Arch Gen Psychiatry 60: 766-775.

Kay SR, Fiszbein A, Opler LA (1987). The positive and negative syndrome scale (PANSS) for schizophrenia. Schizophr Bull 13: 261-276.

Kemether EM, Buchsbaum MS, Byne W, Hazlett EA, Haznedar M, Brickman AM et al (2003). Magnetic resonance imaging of mediodorsal, pulvinar, and centromedian nuclei of the thalamus in patients with schizophrenia. Arch Gen Psychiatry 60: 983-991.

Kraepelin E (1919). Dementia Praecox and Paraphrenia. Edinburgh: E\&S Livingstone.

Kubicki M, Shenton ME, Salisbury DF, Hirayasu Y, Kasai K, Kikinis $\mathrm{R}$ et al (2002). Voxel-based morphometric analysis of gray matter in first episode schizophrenia. Neuroimage 17: 1711-1719.

Kumra S, Giedd JN, Vaituzis AC, Jacobsen LK, McKenna K, Bedwell J et al (2000). Childhood-onset psychotic disorders: magnetic resonance imaging of volumetric differences in brain structure. Am J Psychiatry 157: 1467-1474.

Lieberman JA, Tollefson GD, Charles C, Zipursky R, Sharma T, Kahn RS et al (2005). Antipsychotic drug effects on brain morphology in first-episode psychosis. Arch Gen Psychiatry 62: 361-370.

Mathalon DH, Sullivan EV, Lim KO, Pfefferbaum A (2001). Progressive brain volume changes and the clinical course of schizophrenia in men: a longitudinal magnetic resonance imaging study. Arch Gen Psychiatry 58: 148-157.

Mueser KT, McGurk SR (2004). Schizophrenia. Lancet 363: 20632072.

Pantelis C, Velakoulis D, McGorry PD, Wood SJ, Suckling J, Phillips LJ et al (2003). Neuroanatomical abnormalities before and after onset of psychosis: a cross-sectional and longitudinal MRI comparison. Lancet 361: 281-288.

Pfohl B, Blum N, Zimmerman M (1995). Structured Interview for DSMIV Personality: SIDP-IV. Iowa City: University of Iowa.

Schnack HG, Hulshoff Pol HE, Baare WF, Staal WG, Viergever MA, Kahn RS (2001). Automated separation of gray and white matter from MR images of the human brain. Neuroimage 13: 230-237.

Selemon LD, Mrzljak J, Kleinman JE, Herman MM, Goldman-Rakic PS (2003). Regional specificity in the neuropathologic substrates of schizophrenia: a morphometric analysis of Broca's area 44 and area 9. Arch Gen Psychiatry 60: 69-77.

Selemon LD, Rajkowska G, Goldman-Rakic PS (1995). Abnormally high neuronal density in the schizophrenic cortex. A morphometric analysis of prefrontal area 9 and occipital area 17. Arch Gen Psychiatry 52: 805-818.

Sporn AL, Greenstein DK, Gogtay N, Jeffries NO, Lenane M, Gochman P et al (2003). Progressive brain volume loss during adolescence in childhood-onset schizophrenia. Am J Psychiatry 160: $2181-2189$. 
Thompson PM, Vidal C, Giedd JN, Gochman P, Blumenthal J, Nicolson $\mathrm{R}$ et al (2001). Mapping adolescent brain change reveals dynamic wave of accelerated gray matter loss in very early-onset schizophrenia. Proc Natl Acad Sci USA 98: 1165011655.

Wainer H (1991). Adjusting for differential base rates: Lord's paradox again. Psychol Bull 109: 147-151.

Wood SJ, Velakoulis D, Smith DJ, Bond D, Stuart GW, McGorry $\mathrm{PD}$ et al (2001). A longitudinal study of hippocampal volume in first episode psychosis and chronic schizophrenia. Schizophr Res 52: $37-46$.

Worsley KJ, Marrett S, Neelin P, Vandal AC, Friston KJ, Evans AC (1996). A unified statistical approach for determining significant signals in images of cerebral activation. Hum Brain Mapp 4: 58-73.

Wright IC, Ellison ZR, Sharma T, Friston KJ, Murray RM, McGuire PK (1999). Mapping of grey matter changes in schizophrenia. Schizophr Res 35: 1-14. 